\title{
AN INTEGRAL DUALITY FORMULA
}

\author{
BOGDAN C. GRECU and RAYMOND A. RYAN
}

\begin{abstract}
We establish an integral formula for the duality between multilinear forms/homogeneous polynomials and tensor products for dual spaces with the approximation property and for which the injective tensor products of their preduals is separable and does not contain a copy of $\ell_{1}$. We deduce some multilinear Bishop-Phelps-type results.
\end{abstract}

\section{Introduction}

Although spaces of multilinear forms and homogeneous polynomials are dual spaces, multilinear analogues of the linear Bishop-Phelps Theorem [6] do not hold in general [2], [14]. However, positive results exist. See [5] for a complete account and a detailed description of the problem. For an $n$-linear form defined on a product of Banach spaces $X_{1} \times \cdots \times X_{n}$, two (in general different) types of norm attainment can be considered: as a linear functional defined on the projective tensor product $X_{1} \hat{\otimes}_{\pi} \cdots \hat{\otimes}_{\pi} X_{n}$ or as an $n$-linear form on $X_{1} \times \cdots \times X_{n}$. Under certain conditions, the duality between multilinear forms and tensor products can be expressed by means of an integral formula which will establish the equivalence of the two types of norm attainment and thus yield some multilinear Bishop-Phelps-type results. We note that the same formula, for Hilbert spaces, has already been applied to the study of other geometrical properties of spaces of tensors [11].

\section{Notation and terminology}

(see [10] and [19] for details). Given $n$ (real or complex) Banach spaces $X_{1}, \ldots, X_{n}$, we denote by $X_{1} \otimes \cdots \otimes X_{n}$ their tensor product and by $\pi$ and $\varepsilon$ the projective and injective norms respectively. If $X_{1} \hat{\otimes}_{\pi} \cdots \hat{\otimes}_{\pi} X_{n}$ is the completion of $X_{1} \otimes \cdots \otimes X_{n}$ under the projective norm, then we have $\left(X_{1} \hat{\otimes}_{\pi} \cdots \hat{\otimes}_{\pi} X_{n}\right)^{*}=\mathscr{L}\left({ }^{n} X_{1}, \ldots, X_{n}\right)$, the space of continuous $n$-linear forms on $X_{1} \times \cdots \times X_{n}$ endowed with the supremum norm. We denote by $\mathscr{L}_{I}\left({ }^{n} X_{1}, \ldots, X_{n}\right)=\left(X_{1} \hat{\otimes}_{\varepsilon} \cdots \hat{\otimes}_{\varepsilon} X_{n}\right)^{*}$ and $\mathscr{L}_{N}\left({ }^{n} X_{1}, \ldots, X_{n}\right)$ the spaces of integral and nuclear $n$-linear forms on $X_{1} \times \cdots \times X_{n}$ respectively. Let $\otimes_{n, s} X$

Received June 14, 2005; in revised form October 31, 2005. 
be the $n$-fold symmetric tensor product of $X$. If we endow it with the topology inherited from $X \otimes_{\pi} \cdots \otimes_{\pi} X$ and denote its completion by $\left(\hat{\otimes}_{n, s, \pi} X\right)$ then we have $\left(\hat{\otimes}_{n, s, \pi} X\right)^{*}=\mathscr{L}_{s}\left({ }^{n} X\right)$, the space of continuous symmetric $n$-linear forms on $X \times \cdots \times X$ endowed with the supremum norm.

We denote by $\mathscr{P}\left({ }^{n} X\right)$ the space of all continuous $n$-homogeneous polynomials on $X$ endowed with the natural supremum norm. The space $\otimes_{n, s, \pi} X$ can be renormed such that $\mathscr{P}\left({ }^{n} X\right)$ becomes its dual [10]. Indeed, denote by $x^{n}$ the tensor $x \otimes \cdots \otimes x$. Every element $u$ of $\otimes_{n, s} X$ can be expressed as a finite sum $\sum_{j=1}^{k} \lambda_{j} x_{j}^{n}$. Define the symmetric projective norm of $u$ by

$$
\|u\|_{\pi_{s}}=\inf \left\{\sum_{j=1}^{k}\left|\lambda_{j}\right|\left\|x_{j}\right\|^{n}: u=\sum_{j=1}^{k} \lambda_{j} x_{j}^{n}\right\} .
$$

We denote by $\hat{\otimes}_{n, s, \pi_{s}} X$ the completed tensor product endowed with this norm. Then $\mathscr{P}\left({ }^{n} X\right)$ is the dual space of $\hat{\otimes}_{n, s, \pi_{s}} X$, the duality being given by $\langle u, P\rangle=$ $\sum_{j=1}^{k} \lambda_{j} P\left(x_{j}\right)$.

The symmetric injective norm of $u$ is

$$
\|u\|_{\varepsilon_{s}}=\sup \left\{\left|\sum_{j=1}^{k} \varphi\left(x_{j}\right)^{n}\right|: \varphi \in B_{X^{*}}\right\} .
$$

Because of the polarization formula, the norms $\pi_{s}$ and $\varepsilon_{s}$ on $\otimes_{n, s} X$ are equivalent to $\pi$ and $\varepsilon$ respectively. We have $\left(\hat{\otimes}_{n, s, \varepsilon_{s}} X\right)^{*}=\mathscr{P}_{I}\left({ }^{n} X\right)$, the space of $n$-homogeneous integral polynomials. If the $n$-homogeneous polynomial $P$ is nuclear, then it is integral and $\|P\|_{I} \leq\|P\|_{N}$.

\section{The problem}

Since $\left(\hat{\otimes}_{n, s, \pi_{s}} X\right)^{*}=\mathscr{P}\left({ }^{n} X\right)$, the polynomials that attain their norms on the unit sphere of $\hat{\otimes}_{n, s, \pi_{s}} X$ are a dense set in $\mathscr{P}\left({ }^{n} X\right)$. Say that for a $n$-homogeneous polynomial $P$ on $X$ there exists a tensor $u$ in the unit sphere of $\hat{\otimes}_{n, s, \pi_{s}} X$ such that $\langle u, P\rangle=\|P\|$. Given any $\eta>0$, there exists a sequence $\left(\lambda_{i}\right)$ of scalars with $\sum_{i=1}^{\infty}\left|\lambda_{i}\right|<1+\eta$ and a sequence $\left(x_{i}\right)$ in the unit sphere of $X$ such that $u=\sum_{i=1}^{\infty} \lambda_{i} x_{i}^{n}$ and then $\|P\|=\langle u, P\rangle=\sum_{i=1}^{\infty} \lambda_{i} P\left(x_{i}\right)$. However, for the study of geometrical properties like norm attainment and smoothness, we would like $\sum_{i=1}^{\infty}\left|\lambda_{i}\right|=1=\|u\|_{\pi_{s}}$, as that would imply that $P$ attains its norm on $B_{X}$. This does not happen in general. Under certain hypotheses, we will transform the infinite sum $\sum_{i=1}^{\infty} \lambda_{i} P\left(x_{i}\right)$ into an integral with respect to a measure whose total variation will equal $\|u\|_{\pi_{s}}$, this equality being essential for obtaining norm attainment results. 


\section{Multilinear forms and polynomials defined on dual spaces}

Let $u$ be an element of the completed tensor product $X_{1}^{*} \hat{\otimes}_{\pi} \cdots \hat{\otimes}_{\pi} X_{n}^{*}$. Given any $\eta>0$, there exists sequences $\left(\lambda_{i}\right)$ with $\sum_{i=1}^{\infty}\left|\lambda_{i}\right|<\|u\|_{\pi}+\eta$ and $\left(\varphi_{1, i}\right), \ldots,\left(\varphi_{n, i}\right)$ in the unit spheres of $X_{1}^{*}, \ldots, X_{n}^{*}$ respectively such that

$$
u=\sum_{i=1}^{\infty} \lambda_{i} \varphi_{1, i} \otimes \cdots \otimes \varphi_{n, i} .
$$

We can think of $u$ as a nuclear $n$-linear form on $X_{1} \times \cdots \times X_{n}$, one of its nuclear representations being

$$
u\left(x_{1}, \ldots, x_{n}\right)=\sum_{i=1}^{\infty} \lambda_{i} \varphi_{1, i}\left(x_{1}\right) \cdots \varphi_{n, i}\left(x_{n}\right) .
$$

We can go one step further and consider $u$ as an integral $n$-linear form on $X_{1} \times \cdots \times X_{n}$. In general $\|u\|_{I} \leq\|u\|_{N} \leq\|u\|_{\pi}$. Let $\mu$ be a regular Borel measure on $B_{X_{1}^{*}} \times \cdots \times B_{X_{n}^{*}}$ such that $\|u\|_{I}=\|\mu\|$ and

$$
u\left(x_{1}, \ldots, x_{n}\right)=\int_{B_{X_{1}^{*}} \times \cdots \times B_{X_{n}^{*}}} \varphi_{1}\left(x_{1}\right) \cdots \varphi_{n}\left(x_{n}\right) d \mu\left(\varphi_{1}, \ldots, \varphi_{n}\right) .
$$

In the sequel, each time we will refer to a regular Borel measure on unit balls of dual spaces or their cartesian products we will work with their weak* topologies.

In [3] Alencar showed that $\mathscr{L}_{N}\left({ }^{n} X_{1}, \ldots, X_{n}\right)$ and $\mathscr{L}_{I}\left({ }^{n} X_{1}, \ldots, X_{n}\right)$ are isometrically isomorphic if $X_{1}^{*}, \ldots, X_{n}^{*}$ have the Radon-Nikodým Property (RNP). Furthermore, $\mathscr{L}_{I}\left({ }^{n} X_{1}, \ldots, X_{n}\right)$ has RNP [18]. Thus $X_{1} \hat{\otimes}_{\varepsilon} \cdots \hat{\otimes}_{\varepsilon} X_{n}$ cannot contain a copy of $\ell_{1}$. Alencar's result can be extended to all spaces $X_{1}, \ldots, X_{n}$ with the property that $X_{1} \hat{\otimes}_{\varepsilon} \ldots \hat{\otimes}_{\varepsilon} X_{n}$ does not contain a copy of $\ell_{1}$, using the fact that the set of extreme points of the unit ball of $\mathscr{L}_{I}\left({ }^{n} X_{1}, \ldots\right.$, $X_{n}$ ) is equal to $\left\{\varphi_{1} \cdots \varphi_{n}: \varphi_{i} \in \operatorname{Ext} B_{X_{i}^{*}}\right\}$ (Theorem 1.1 of [18]) and following the reasoning in Proposition 3 of [7].

THEOREM 1. If $X_{1} \hat{\otimes}_{\varepsilon} \cdots \hat{\otimes}_{\varepsilon} X_{n}$ is separable and does not contain a copy of $\ell_{1}$ and at least $n-1$ of the duals $X_{1}^{*}, \ldots, X_{n}^{*}$ have the approximation property $(A P)$, then for every element $u$ of $X_{1}^{*} \hat{\otimes}_{\pi} \cdots \hat{\otimes}_{\pi} X_{n}^{*}$, there exists a regular Borel measure $\mu$ on $B_{X_{1}^{*}} \times \cdots \times B_{X_{n}^{*}}$ such that $\|u\|_{\pi}=\|\mu\|$ and

$$
\langle u, A\rangle=\int_{B_{X_{1}^{*}} \times \cdots \times B_{X_{n}^{*}}} A\left(\varphi_{1}, \ldots, \varphi_{n}\right) d \mu\left(\varphi_{1}, \ldots, \varphi_{n}\right)
$$

for every continuous $n$-linear form $A$ on $X_{1}^{*} \times \cdots \times X_{n}^{*}$. 
Proof. Due to the fact that at least $n-1$ of the duals $X_{1}^{*}, \ldots, X_{n}^{*}$ have AP, the spaces $X_{1}^{*} \hat{\otimes}_{\pi} \cdots \hat{\otimes}_{\pi} X_{n}^{*}$ and $\mathscr{L}_{N}\left({ }^{n} X_{1}, \ldots, X_{n}\right)$ are isometrically isomorphic and so

$$
\begin{aligned}
\mathscr{L}\left({ }^{n} X_{1}^{*}, \ldots, X_{n}^{*}\right) & =\left(X_{1}^{*} \hat{\otimes}_{\pi} \cdots \hat{\otimes}_{\pi} X_{n}^{*}\right)^{*}=\mathscr{L}_{N}\left({ }^{n} X_{1}, \ldots, X_{n}\right)^{*} \\
& =\mathscr{L}_{I}\left({ }^{n} X_{1}, \ldots, X_{n}\right)^{*}=\left(X_{1} \otimes_{\varepsilon} X_{2} \otimes_{\varepsilon} \cdots \otimes_{\varepsilon} X_{n}\right)^{* *} .
\end{aligned}
$$

Thus, by the Odell-Rosenthal Theorem [16], every multilinear form $A$ on $X_{1}^{*} \times \cdots \times X_{n}^{*}$ is the weak ${ }^{*}$-limit of a sequence $\left(v_{n}\right)$ from $X_{1} \otimes_{\varepsilon} X_{2} \otimes_{\varepsilon} \cdots \otimes_{\varepsilon} X_{n}$ with $\left\|v_{n}\right\|_{\varepsilon} \leq\|A\|$. Let $\mu$ be a regular Borel measure on $B_{X_{1}^{*}} \times \cdots \times B_{X_{n}^{*}}$ such that $\|\mu\|=\|u\|_{I}=\|u\|_{N}=\|u\|_{\pi}$. Since every $v_{n}$ is a linear combination of basic tensors, we have

$$
\left\langle u, v_{n}\right\rangle=\int_{B_{X_{1}^{*} \times \cdots \times B_{X_{n}^{*}}}} v_{n}\left(\varphi_{1}, \ldots, \varphi_{n}\right) d \mu\left(\varphi_{1}, \ldots, \varphi_{n}\right)
$$

and thus

$$
\begin{aligned}
\langle u, A\rangle=\lim _{n}\left\langle u, v_{n}\right\rangle & =\lim _{n} \int_{B_{X_{1}^{*} \times \cdots \times B_{X_{n}^{*}}}} v_{n}\left(\varphi_{1}, \ldots, \varphi_{n}\right) d \mu\left(\varphi_{1}, \ldots, \varphi_{n}\right) \\
& =\int_{B_{X_{1}^{*}} \times \cdots \times B_{X_{n}^{*}}} A\left(\varphi_{1}, \ldots, \varphi_{n}\right) d \mu\left(\varphi_{1}, \ldots, \varphi_{n}\right),
\end{aligned}
$$

by the Dominated Convergence Theorem.

Remark. It follows from the proof of the theorem that the function $J$, defined on $B_{X_{1}^{*}} \times \cdots \times B_{X_{n}^{*}}$ with values in $X_{1}^{*} \hat{\otimes}_{\pi} \cdots \hat{\otimes}_{\pi} X_{n}^{*}$ by $\left(\varphi_{1}, \ldots, \varphi_{n}\right) \stackrel{J}{\mapsto}$ $\varphi_{1} \otimes \cdots \otimes \varphi_{n}$, is weakly $\mu$-measurable and that the tensor $u$ can be expressed as a Pettis integral

$$
u=\int_{B_{X_{1}^{*} \times \cdots \times B_{X_{n}^{*}}}} \varphi_{1} \otimes \cdots \otimes \varphi_{n} d \mu\left(\varphi_{1}, \ldots, \varphi_{n}\right) .
$$

Since $\|\mu\|=\|u\|_{\pi}<\infty$, the function $J$ is Bochner integrable with respect to $\mu$ whenever it is $\mu$-measurable. By the Pettis Measurability Theorem [9], this will happen when we work under the additional hypothesis that all the spaces $X_{1}^{*}, \ldots, X_{n}^{*}$ are separable, in which case the integral above will actually be a Bochner integral.

Now suppose that the $n$-linear form $A$, as a linear functional on $X_{1}^{*} \hat{\otimes}_{\pi} \cdots$ 
$\hat{\otimes}_{\pi} X_{n}^{*}$, attains its norm at a tensor $u$ of unit norm. Then

$$
\begin{aligned}
\|A\| & =\langle u, A\rangle=\int_{B_{X_{1}^{*} \times \cdots \times B_{X_{n}^{*}}} A\left(\varphi_{1}, \ldots, \varphi_{n}\right) d \mu\left(\varphi_{1}, \ldots, \varphi_{n}\right)} \\
& \leq\|A\|\|\mu\|=\|A\| .
\end{aligned}
$$

It follows that $A\left(\varphi_{1}, \ldots, \varphi_{n}\right)=\|A\|$ a.e. with respect to $\mu$ and so $A$ attains its norm as an $n$-linear form. Applying the Bishop-Phelps Theorem for $\left(X_{1}^{*} \hat{\otimes}_{\pi} \cdots \hat{\otimes}_{\pi} X_{n}^{*}\right)^{*}$, we obtain the following

CoRollaRy 2. If $X_{1} \hat{\otimes}_{\varepsilon} \cdots \hat{\otimes}_{\varepsilon} X_{n}$ is separable and does not contain a copy of $\ell_{1}$ and at least $n-1$ of the duals $X_{1}^{*}, \ldots, X_{n}^{*}$ have AP then the set of norm-attaining $n$-linear forms on $X_{1}^{*} \times \cdots \times X_{n}^{*}$ is dense in $\mathscr{L}\left({ }^{n} X_{1}^{*}, \ldots, X_{n}^{*}\right)$.

In particular the result holds if the non-containment of $\ell_{1}$ in $X_{1} \hat{\otimes}_{\varepsilon} \cdots \hat{\otimes}_{\varepsilon} X_{n}$ is replaced by the stronger condition that $X_{1}^{*}, \ldots, X_{n}^{*}$ have RNP. This can be obtained in an alternative way, since under these conditions $X_{1}^{*} \hat{\otimes}_{\pi} \cdots \hat{\otimes}_{\pi} X_{n}^{*}$ has RNP [9] and norm-attaining linear functionals on spaces with RNP attain their norms at extreme points, which, as noted above, are of the form $\varphi_{1} \otimes$ $\cdots \otimes \varphi_{n}$ with $\varphi_{i}$ extreme points of $B_{X_{i}^{*}}$. However there exists separable spaces whose duals do not have RNP which satisfy the conditions of the theorem. One such example is the James-Hagler space $J H$ [13]. Its injective tensor product does not contain a copy of $\ell_{1}$ [15], its dual does not have RNP but does have AP. This last fact follows from Lemma 7.3.c in [8], since $J H$ has Schauder basis and for $F$, the closure in $J H^{*}$ of the subspace generated by the coefficient functionals, the quotient $J H^{*} / F$ is isometrically isomorphic to a $c_{0}$ space.

In [4] a variational principle due to Stegall is used to obtain the density in $\mathscr{L}\left({ }^{n} X\right)$ of the set of norm-attaining $n$-linear forms defined on a Banach space $X$ with RNP. However, the proof is highly non-symmetrical, so no result is obtained for symmetric $n$-linear forms. The advantage of the integral formula is that it allows us to obtain multilinear Bishop-Phelps-type results for any spaces of multilinear forms that are duals of corresponding spaces of tensors, e.g. symmetric, alternating, Jacobian, etc. [12]. For instance, we have:

Corollary 3. Let $X$ be a separable Banach space such that $X^{*}$ has AP and $\hat{\otimes}_{n, \varepsilon} X$ does not contain a copy of $\ell_{1}$. Then the set of norm-attaining symmetric $n$-linear forms on $X^{*}$ is dense in $\mathscr{L}_{s}\left({ }^{n} X^{*}\right)$.

As above, if $X^{*}$ has RNP, the result can be obtained in an alternative way, due to the fact that the set of extreme points of the unit ball of $\hat{\otimes}_{n, s, \pi} X^{*}$ is a subset of $\left\{\varphi_{1} \otimes_{s} \cdots \otimes_{s} \varphi_{n}:\left\|\varphi_{i}\right\|=1\right\}$ (by the same reasoning as in proposition 1 of [7]). 
In the light of this fact, repeating line by line the proof of the theorem, but working with symmetric $n$-linear forms and tensors, Corollary 3 remains valid under the slightly weaker condition that $\hat{\otimes}_{n, s, \varepsilon} X$ does not contain a copy of $\ell_{1}$.

A similar integral formula holds for $n$-homogeneous polynomials. In [11] this formula has been obtained for the case $n=2$ for Hilbert spaces.

THEOREM 4. If $\hat{\otimes}_{n, s, \varepsilon_{s}} X$ is separable and does not contain a copy of $\ell_{1}$ and $X^{*}$ has AP, then for every element u of $\hat{\otimes}_{n, s, \pi_{s}} X^{*}$ there exists a regular Borel measure $\mu$ on $B_{X^{*}}$ such that $\|u\|_{\pi_{s}}=\|\mu\|$ and

$$
\langle u, P\rangle=\int_{B_{X^{*}}} P(\varphi) d \mu(\varphi)
$$

for every $n$-homogeneous polynomial $P$ on $X^{*}$.

The proof mimics that of Theorem 1 , using the fact that every element $u$ of $\hat{\otimes}_{n, s, \pi_{s}} X^{*}$ can be considered as a nuclear, and thus integral, polynomial on $X$ and the measure $\mu$ on $B_{X^{*}}$ associated with $u$ can be chosen such that $\|\mu\|=\|u\|_{I} \leq\|u\|_{N} \leq\|u\|_{\pi_{s}}$. Then $\mathscr{P}_{N}\left({ }^{n} X\right)=\mathscr{P}_{I}\left({ }^{n} X\right)$ when $\hat{\otimes}_{n, s, \varepsilon_{s}} X$ does not contain a copy of $\ell_{1}$ [7] and $\mathscr{P}_{N}\left({ }^{n} X\right)=\hat{\otimes}_{n, s, \pi_{s}} X^{*}$ when $X^{*}$ has AP, which give

$$
\mathscr{P}\left({ }^{n} X^{*}\right)=\left(\hat{\otimes}_{n, s, \pi_{s}} X^{*}\right)^{*}=\mathscr{P}_{N}\left({ }^{n} X\right)^{*}=\mathscr{P}_{I}\left({ }^{n} X\right)^{*}=\left(\hat{\otimes}_{n, s, \varepsilon_{s}} X\right)^{* *} .
$$

Let $\mathscr{P}_{w^{*}}\left({ }^{n} X^{*}\right)$ be the space of $n$-homogeneous polynomials on $X^{*}$ that are $w^{*}$-continuous on bounded subsets of $X^{*}$. In [21] it is shown that $\mathscr{P}\left({ }^{n} X^{*}\right)$ coincides with the bidual of $\mathscr{P}_{w^{*}}\left({ }^{n} X^{*}\right)$ whenever $X^{*}$ has AP and $\mathscr{P}_{w^{*}}\left({ }^{n} X^{*}\right)$ does not contain a copy of $\ell_{1}$. We have just obtained another proof of this result (under the assumption of separability) since $\mathscr{P}_{w^{*}}\left({ }^{n} X^{*}\right)=\hat{\otimes}_{n, s, \varepsilon_{s}} X$ whenever $X$ has AP.

Corollary 5. Let $X$ be a separable Banach space such that $X^{*}$ has $A P$ and $\hat{\otimes}_{n, s, \varepsilon_{s}} X$ does not contain a copy of $\ell_{1}$. The set of norm-attaining $n$-homogeneous polynomials on $X^{*}$ is dense in $\mathscr{P}\left({ }^{n} X^{*}\right)$.

A natural question is whether the integral formulae above can be obtained from Choquet's Theorem [17]. Let us work under the assumptions of Theorem 4 . Then the unit ball of $\hat{\otimes}_{n, s, \pi_{s}} X^{*}$ is weak*-compact. The Choquet Theorem says that, given $u$ in $B_{\hat{\otimes}_{n, s, \pi_{s}} X^{*}}$, there exists a probability measure $\mu$ supported by the weak*-closure of the extreme points of $B_{\hat{\otimes}_{n, s, \pi_{s}} X^{*}}$ such that

$$
\langle u, v\rangle=\int_{B_{\hat{\otimes}_{n, s, \pi_{S}} X^{*}}}\langle w, v\rangle d \mu(w)
$$


for all $v$ in $\left(\hat{\otimes}_{n, s, \pi_{s}} X^{*}, w^{*}\right)^{*}=\hat{\otimes}_{n, s, \varepsilon_{s}} X$. Furthermore, the set of extreme points of $B_{\hat{\otimes}_{n, s, \pi_{s}} X^{*}}$ is included in $\{\varphi \otimes \cdots \otimes \varphi:\|\varphi\|=1\}$ and includes $\{\varphi \otimes \cdots \otimes \varphi:\|\varphi\|=1$ and $\varphi$ is norm-attaining $\}$ [7]. However, the weak* closure of this set can be quite large, although $\{\varphi \otimes \cdots \otimes \varphi:\|\varphi\|=1\}$ is norm-closed or even weakly-closed in particular cases [20]. For instance, if $X$ is a Hilbert space then its $w^{*}$-closure is the whole of $B_{\hat{\otimes}_{n, s, \pi_{s}} X^{*}}$. Thus there is no reason to believe that $\mu$ is supported by $\{\varphi \otimes \cdots \otimes \varphi:\|\varphi\|=1\}$, a fact that was essential in deducing the Bishop-Phelps-type results.

ACKNOWLEDGMENTS. Both authors wish to acknowledge the financial support of an Enterprise Ireland Basic Research Grant. Part of this work was done while the first author visited the Department of Mathematical Analysis of the University of Valencia, Spain, which hospitality is gratefully acknowledged.

\section{REFERENCES}

1. Acosta, M. D., On multilinear mappings attaining their norms, Studia Math. 131 (1998), no. 2, 155-165.

2. Acosta, M. D., Aguirre, F. J., Payá, R., There is no bilinear Bishop-Phelps theorem, Israel J. Math. 93 (1996), 221-227.

3. Alencar, R., Multilinear mappings of nuclear and integral type, Proc. Amer. Math. Soc. 94 (1985), no. 1, 33-38.

4. Aron, R. M., Finet, C., Werner, E., Some remarks on norm-attaining $n$-linear forms, Function spaces (Edwardsville, IL, 1994), 19-28, Lecture Notes in Pure and Appl. Math. 172 (1995).

5. Aron, R. M., García, D., Maestre, M., On norm attaining polynomials, Publ. Res. Inst. Math. Sci. 39 (2003), no. 1, 165-172.

6. Bishop, E., Phelps, R. R., A proof that every Banach space is subreflexive, Bull. Amer. Math. Soc. 67 (1961), 97-98.

7. Boyd, C., Ryan, R. A., Geometric theory of spaces of integral polynomials and symmetric tensor products, J. Funct. Anal. 179 (2001), 18-42.

8. Castillo, J. M. F., González, M., Three-space Problems in Banach Space Theory, Lecture Notes in Math. 1667 (1997).

9. Diestel, J., Uhl, J. J. Jr., Vector Measures, Math. Surveys Monogr. 15 (1977).

10. Dineen, S., Complex Analysis on Infinite Dimensional Spaces, Springer Monogr. Math. (1999).

11. García, D., Grecu, B. C., Maestre, M., Smoothness in preduals of spaces of polynomials, preprint.

12. Grecu, B. C., Ryan, R. A., Tensor products of direct sums, Ark. Mat. 43 (2005), no. 1, 167-180.

13. Hagler, J., A counterexample to several questions about Banach spaces, Studia Math. 60 (1977), no. 3, 289-308.

14. Jiménez Sevilla, M., Payá, R., Norm attaining multilinear forms and polynomials on preduals of Lorentz sequence spaces, Studia Math. 127 (1998), no. 2, 99-112.

15. Leung, D. H., Embedding $l^{1}$ into tensor products of Banach spaces, Functional analysis (Austin, TX, 1987/1989), 171-176, Lecture Notes in Math. 1470 (1991).

16. Odell, E., Rosenthal, H. P., A double-dual characterization of separable Banach spaces containing $l^{1}$, Israel J. Math. 20 (1975), no. 3-4, 375-384.

17. Phelps, R. R., Lectures on Choquet's theorem, 2nd ed., Lecture Notes in Math. 1757 (2001). 
18. Ruess, W. M., Stegall, C. P., Extreme points in duals of operator spaces, Math. Ann. 261 (1982), no. 4, 535-546.

19. Ryan, R. A., Introduction to Tensor Products of Banach Spaces, Springer Monogr. Math. (2002).

20. Ryan, R. A., Turett, B., Geometry of spaces of polynomials, J. Math. Anal. Appl. 221 (1998), 698-711.

21. Valdivia, M., Banach spaces of polynomials without copies of $l^{1}$, Proc. Amer. Math. Soc. 123 (1995), no. 10, 3143-3150.

MATHEMATICS DEPARTMENT

NATIONAL UNIVERSITY OF IRELAND

GALWAY

IRELAND

E-mail: bogdan@wuzwuz.nuigalway.ie, ray.ryan@nuigalway.ie 